\title{
LECCIONES GLOBALES DE LA REFORMA MEXICANA DE SALUD: EMPODERAMIENTO A TRAVÉS DEL USO DE EVIDENCIAS
}

\author{
Julio Frenk $k^{1, a, b, c, d}$, Octavio Gómez-Dantés 2 ,a,b
}

\begin{abstract}
RESUMEN
En este artículo se ilustra, con la reciente reforma al sistema mexicano de salud, el potencial del conocimiento en el diseño e implantación de las políticas públicas. En primer lugar se discute la relación entre conocimiento y salud. En una segunda parte se describen los esfuerzos que se llevaron a cabo en México para generar evidencias que eventualmente nutrieron el diseño e implantación de las políticas locales de salud. En seguida se analizan los contenidos de la reforma del sistema mexicano de salud y su concepto rector, La democratización de la atención a la salud. El artículo concluye con una discusión de las lecciones globales de esta experiencia de reforma.
\end{abstract}

Palabras clave: Reforma de la atención en salud; Bases del conocimiento; Salud pública; México (fuente: DeCS BIREME).

\section{GLOBAL LESSONS OF THE MEXICAN HEALTH REFORM: EMPOWERMENT THROUGH THE USE OF EVIDENCE}

\begin{abstract}
This paper illustrates, using as an example the recent reform of the Mexican health system, the potential of knowledge in the design and implementation of public policies. In the first part the relationship between knowledge and health is described. In part two, the efforts in Mexico to generate evidence that would eventually nourish the design and implementation of health policies are discussed. In the following sections the content and the guiding concept of the reform, the democratization of health, are analyzed. The paper concludes with the discussion of the main global lessons of this reform experience.
\end{abstract}

Key words: Health care reform; Knowledge bases; Public Health; Mexico (source: MeSH NLM).

\section{INTRODUCCIÓN}

La salud ocupa ya un lugar central en las principales dimensiones de la agenda global: la seguridad, el desarrollo económico, la gobernanza democrática y los derechos humanos. Este reconocimiento se ha asociado con una expansión sin precedentes de la asistencia para el desarrollo destinada a la salud, que se incrementó de 10 mil millones de dólares en 2000 a casi 22 mil millones

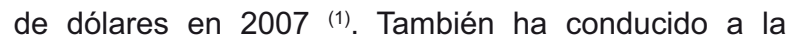
creación de nuevas formas de organización. Hoy en día, hay cerca de 120 agencias y asociaciones multilaterales activas en el campo de la salud.

Para enfrentar los retos y aprovechar las oportunidades de un mundo cada vez más interdependiente, es indispensable renovar la cooperación global en salud. En este proceso de renovación, la generación, uso y diseminación del conocimiento cumplen un papel crítico.
El principal mensaje de este artículo es que las políticas basadas en evidencias no son simple retórica sino una posibilidad real. El potencial del conocimiento en el diseño e implantación de las políticas públicas se ilustra con la reciente reforma del sistema mexicano de salud, que puede considerarse un caso de libro de texto de una política de salud basada en evidencias. En la primera parte se discute la relación entre conocimiento y salud. En seguida se describen los esfuerzos que se llevaron a cabo en México para generar evidencias que, eventualmente, nutrieron el diseño e implantación de las políticas locales de salud. En la tercera y cuarta parte se discute los contenidos de la reforma del sistema mexicano de salud y su concepto rector, La democratización de la atención a la salud. La quinta parte se dedica a describir las evaluaciones a las que ha sido sujeta esta política pública. El artículo concluye con la discusión de algunas lecciones globales de esta experiencia de reforma.

\footnotetext{
1 Dean, Harvard School of Public Health, Harvard University. Boston, USA.

2 Instituto Nacional de Salud Pública. Cuernavaca, México.

a Médico; b Magíster en Salud Pública; ' Magíster en Sociología; d Doctor (PhD) en Organización del Cuidado Médico y Sociología.
}

\begin{tabular}{|ll|}
\hline Recibido: 01-06-10 Aprobado: 18-08-10 \\
\hline
\end{tabular}




\section{CONOCIMIENTO Y SALUD}

En el siglo XX el mundo experimentó mayores ganancias en la esperanza de vida que en toda la historia acumulada de la humanidad. La esperanza de vida al nacer era de 30 años en 1900. En 2008 alcanzó la cifra de 68,8 años, aunque con grandes diferencias regionales: 82 años en Japón y apenas 32 años en Swazilandia ${ }^{(2)}$. Ahora sabemos que la mayor parte de estas mejoras se dieron como resultado de lo que Deaton llama la globalización del conocimiento relacionado con la salud (3).

Hay tres mecanismos básicos a través de los cuales el conocimiento mejora la salud (Figura 1). En primer lugar, el conocimiento se traduce en nuevas tecnologías, como vacunas, medicamentos y métodos diagnósticos. En segundo lugar, el conocimiento es internalizado por los individuos para estructurar su vida diaria en dominios tan críticos como la higiene personal, los hábitos alimenticios, la sexualidad y la crianza de los hijos. Finalmente, el conocimiento se traduce en evidencias que sustentan el diseño e implantación de las políticas públicas.

En este artículo se describe un ejemplo exitoso de uso del conocimiento en el diseño, implantación y evaluación de una política pública: la reciente reforma al sistema mexicano de salud, cuyo propósito es extender la protección social en salud a toda la población.

\section{EVIDENCIAS PARA EL DISEÑO DE POLÍTICAS DE SALUD EN MÉXICO}

Los instrumentos analíticos para el diseño e implantación de políticas de salud se han enriquecido considerablemente en los últimos años. Destacan dentro de ellos la metodología para calcular la llamada carga de la enfermedad, el análisis de la relación costo-efectividad de las intervenciones de salud y las cuentas nacionales de salud.

La aplicación de estos bienes públicos globales relacionados con el conocimiento, ayudó a catalizar una reforma estructural del sistema mexicano de salud a principios de esta década.

El uso local de estos bienes públicos globales está íntimamente relacionado con la creación de dos instituciones que han fortalecido las capacidades analíticas, gerenciales y de abogacía en México, en el campo de la salud pública: la Fundación Mexicana para la Salud (FMS), creada en 1985, y el Instituto Nacional de Salud Pública (INSP), creado en 1987.

A principios de los años noventa, diversos investigadores de estas dos instituciones, en colaboración con investigadores de la Escuela de Salud Pública de la Universidad Harvard, midieron por vez primera la carga de la enfermedad en México ${ }^{(4)}$. Utilizaron para ese fin el indicador "Años de vida saludables perdidos" (AVISA), que suma los años de vida saludable que se pierden tanto por muerte prematura como por discapacidad. La gran utilidad de este tipo de indicadores es que permite identificar necesidades de salud, como las enfermedades mentales, que no suelen recibir la atención requerida porque no conducen a la muerte.

Los resultados de este estudio confirmaron lo que algunos de esos mismos investigadores habían planteado unos años antes ${ }^{(5)}$, la existencia en México de un doble reto en materia de salud: i) terminar con el rezago representado por las llamadas enfermedades del subde-

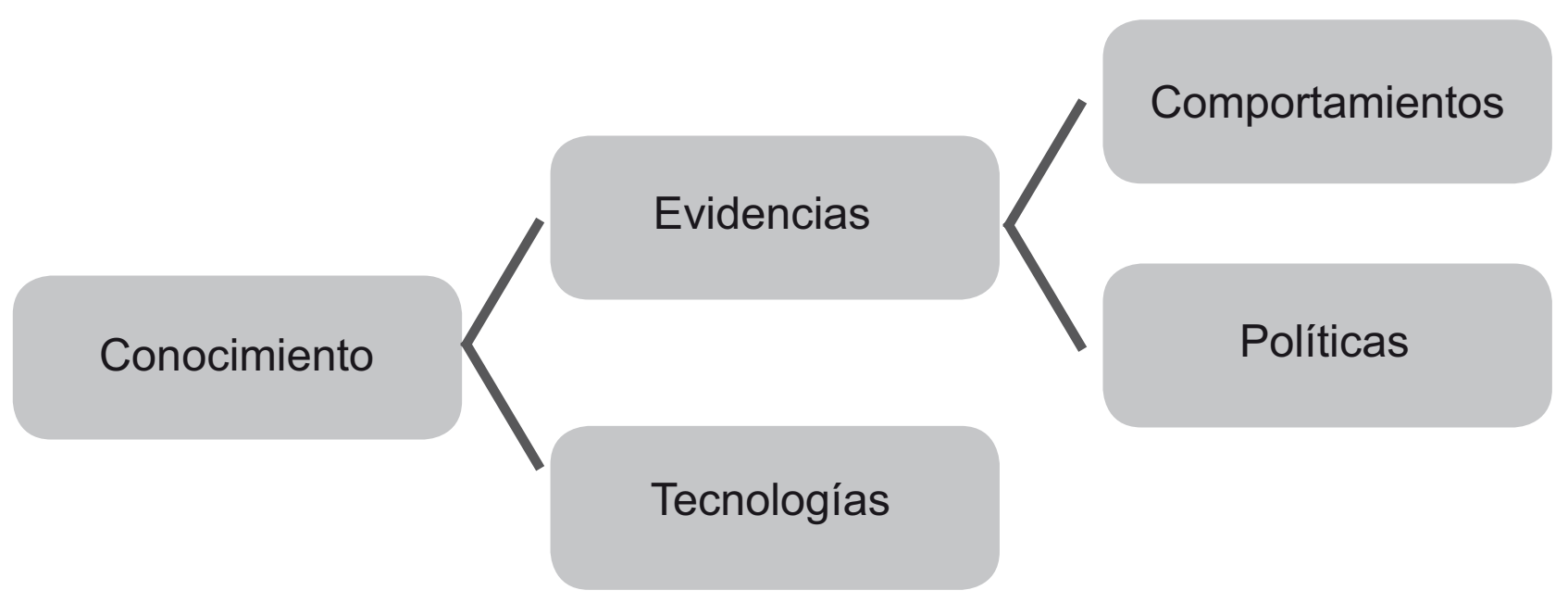

Figura 1. Traducción del conocimiento en evidencias y tecnologías que mejoran la salud. 
sarrollo, como las infecciones comunes, la desnutrición, y las muertes maternas y perinatales, y ii) enfrentar los problemas emergentes asociados con la urbanización y la industrialización, como las enfermedades cardiovasculares, la diabetes, el cáncer, las enfermedades mentales y las lesiones, que ya dominaban el perfil epidemiológico del país.

Por su parte, el cálculo de las cuentas nacionales de salud, que la FMS realizó también a principios de la última década del siglo pasado, reveló dos hechos muy importantes: que el gasto en salud en México era insuficiente para atender los retos planteados por la transición epidemiológica y que más de la mitad del gasto total en salud en México era privado ${ }^{(6)}$.

A principios de los noventa México destinaba a la salud alrededor del $5 \%$ de su PIB, cifra inferior al promedio latinoamericano de $1990(5,7 \%)$ y muy inferior a la cifra de otros países de ingresos medios de la región. El grueso de este pobre gasto en salud, además, era privado, en su mayor parte, gasto de bolsillo. Esto último era resultado directo de que aproximadamente la mitad de la población, alrededor de 50 millones de personas, carecía de seguro de salud.

La alta prevalencia del gasto de bolsillo exponía a los hogares mexicanos a incurrir en gastos excesivos para atender sus necesidades de salud. De hecho, por esta razón, México se desempeñó muy mal en el análisis comparativo internacional sobre financiamiento justo que la Organización Mundial de la Salud llevó a cabo en el año $2000{ }^{(7)}$.

En lugar de generar una reacción defensiva, estos resultados convencieron al Ministerio de Salud (MS) de solicitar análisis adicionales que demostraron que los gastos excesivos por motivos de salud en México afectaban a $4 \%$ de los hogares mexicanos, y que eran mucho más frecuentes en los hogares pobres no asegurados ${ }^{(8)}$.

Otros análisis permitieron documentar la existencia de tres desequilibrios financieros más: de distribución, de esfuerzo estatal y de destino de los recursos ${ }^{(9)}$.

La segmentación del sistema mexicano de salud había favorecido la concentración de los recursos públicos para la salud en las instituciones de seguridad social. El gobierno federal asignaba 1,5 veces más recursos per cápita a la población asegurada que a la población no asegurada. A esto se sumaba la inequitativa distribución de los recursos federales entre entidades federativas.

El indiscutible avance que representó la progresiva descentralización de los servicios de salud, que dio inicio a principios de los ochenta y culminó en el año 2000, no se acompañó de reglas claras que estipularan la corresponsabilidad de los distintos niveles de gobierno. Esta indefinición se reflejaba en las enormes diferencias que se registraban entre los estados en materia de contribución estatal a la salud. Había gobiernos estatales que, en 2001, asignaban a la salud hasta 119 veces más recursos per cápita que las entidades federativas que menos contribuían con recursos propios a este sector.

El último de los desequilibrios se refiere al destino de los recursos. El sector público de la salud dedicaba cada vez más dinero al pago de los salarios y cada vez menos recursos al gasto de inversión, hecho que influía negativamente en la ampliación de la infraestructura y el equipamiento de las unidades.

\section{LA REFORMA DEL SISTEMA DE SALUD DE MÉXICO}

Estas evidencias, que documentaban los grandes retos que enfrentaba el país en materia de salud, facilitaron la generación de instrumentos de abogacía que permitieron promover una reforma legislativa que dio origen, en 2003, al Sistema de Protección Social en Salud (SPSS), cuyo brazo operativo es el Seguro Popular de Salud (SPS).

En este ensayo no se discutirán los detalles de la reforma mexicana, que se describen exhaustivamente en una serie de siete artículos publicados en la revista Lancet ${ }^{(10-16)}$. Baste con señalar que el SPSS está reorganizando el gasto público en salud y ampliándolo en un punto porcentual del PIB en un periodo de siete años para proporcionar protección social en salud a toda la población, incluyendo los 50 millones de mexicanos que habían sido excluidos de la seguridad social convencional por ser auto-empleados, estar fuera del mercado de trabajo o trabajar en el sector informal de la economía.

Las familias pobres pueden ahora afiliarse al SPS, que ofrece el acceso a 266 intervenciones incluidas en el Catálogo Universal de Servicios de Salud (CAUSES) que cubren más del $90 \%$ de la demanda de atención en las unidades ambulatorias y los hospitales generales públicos. Además, los afiliados a este seguro tienen acceso a 49 intervenciones que generan gastos catastróficos, incluyendo el tratamiento para cáncer en niños, VIH/SIDA, cáncer cérvico-uterino y cáncer de mama, entre otros.

Esta reforma financiera se complementó con una reforma gerencial que comprende la implantación de un Plan Maestro de Infraestructura Física; la acreditación de uni- 
dades; la certificación de proveedores de servicios; el diseño de programas de garantía de calidad; mejoras en el abasto de medicamentos y otros insumos; el fortalecimiento de los sistemas de información en salud y la evaluación comparativa del desempeño de los sistemas estatales e institucionales de salud.

A la fecha, se han afiliado al SPS 10,5 millones de familias, que representan 31,1 millones de beneficiarios (17). Poco más de $95 \%$ de estas familias se ubican en los dos primeros deciles de ingreso y $35 \%$ habitan en zonas rurales.

\section{LA DEMOCRATIZACIÓN DE LA ATENCIÓN A LA SALUD}

La reforma mexicana se diseñó e implantó haciendo uso de lo que Reich identifica como los tres pilares de las políticas públicas: el pilar técnico, el pilar político y el pilar ético ${ }^{(18)}$. Estos tres pilares están estrechamente vinculados. En particular, los arreglos políticos, que son indispensables para el éxito de una reforma, solo pueden alcanzarse cuando los guían las ideas y los ideales ${ }^{(19)}$. Las ideas proceden del conocimiento que genera la ciencia. Los ideales proceden de los valores que derivan de la ética.

El pilar técnico de la reforma se construyó, como ya se mencionó, haciendo uso intensivo de evidencias sólidas derivadas de la adaptación local de bienes públicos globales relacionados con el conocimiento. Estas evidencias empoderaron a los tomadores de decisiones con medios para confrontar el status quo y promover el cambio.

Alcanzar consensos en medio de los fragores de una joven democracia fue posible también gracias al uso de deliberaciones sobre las implicaciones morales de los arreglos existentes que, como ya se señaló, excluían de la protección social en salud a la mitad de la población. La premisa de la que se partió fue muy clara: todos los sistemas de salud reflejan una serie de supuestos éticos. Explícita o implícitamente, estos supuestos se expresan en la distribución de beneficios de atención a la salud y en la organización de las instituciones. Junto con la formulación de propuestas técnicas y estrategias políticas, cada intento por reformar el sistema de salud debe empezar por preguntarse sobre los valores que se han de promover ${ }^{(20)}$. De esta manera, los fundamentos éticos de las propuestas de reforma pueden hacerse explícitos y transparentes ${ }^{(21,22)}$.

La reforma mexicana se formuló y promovió sobre la base un concepto rector, la "democratización de la salud", que involucra la expansión de la democracia al ámbito de los derechos sociales ${ }^{(23)}$.

Según O’Donnel y Schmitter, la "democratización" supone la aplicación de las normas y los procedimientos de la ciudadanía a instituciones que estaban regidas por otros principios, como el control coactivo, la tradición social, el juicio de los especialistas o las prácticas administrativas ${ }^{(24)}$.

En su obra seminal sobre Clase, ciudadanía y desarrollo social, T.H. Marshall identifica tres tipos de derechos constitutivos de la ciudadanía: los derechos civiles, los derechos políticos y los derechos sociales ${ }^{(25)}$. De acuerdo con Marshall, la ciudadanía culmina con el ejercicio efectivo de los derechos sociales.

Como resultado de su proceso democratizador, México había avanzado considerablemente en el ejercicio de los derechos políticos y civiles, pero era claro que el siguiente gran reto era abatir las desigualdades asegurando el ejercicio universal de los derechos sociales, incluyendo el derecho a la protección de la salud.

Aunque el derecho a la protección de la salud estaba reconocido en la Constitución mexicana desde 1983, no todos los individuos lo habían podido ejercer por igual. La mitad de la población, que contaba con empleo formal, disfrutaba de seguridad social. La otra mitad, sin embargo, había quedado marginada de todo tipo de seguro de salud.

Así que el reconocimiento formal de la atención a la salud como un derecho social estaba allí cuando la reforma empezó a discutirse, pero su ejercicio real estaba limitado solo a un sector de la población. Lo que hacía falta era la identificación de los beneficios concretos que derivaban de dicho derecho, y el diseño de los vehículos financieros y organizacionales para traducirlos en servicios de salud efectivos para todos.

De acuerdo con Brachet, la transformación de la atención a la salud en un derecho social exige: i) la definición de un conjunto de intervenciones a las que todos los ciudadanos, independientemente de su condición socioeconómica o estatus laboral, tienen derecho y pueden demandar legalmente, y ii) la definición de la forma en que el costo de este conjunto de servicios habrá de repartirse entre los diferentes sectores de la población a fin de asegurar su viabilidad financiera ${ }^{(26)}$.

Las reformas a la Ley General de Salud (LGS) aprobadas en 2003 señalan que los afiliados al SPS tendrán acceso a todas las intervenciones incluidas en el CAUSES y todas las intervenciones financiadas con el Fondo de 
Protección contra Gastos Catastróficos. De hecho, en el momento de afiliación, los beneficiarios de este seguro reciben la Carta de Derechos y Obligaciones de los Afiliados al SPS, que contiene la lista de intervenciones a las que por ley tienen derecho.

La LGS también estipula que las intervenciones del CAUSES deben ampliarse conforme se disponga de nuevas tecnologías y recursos, pero también conforme se identifiquen nuevos padecimientos y factores de riesgo. Los servicios cubiertos se analizan y eligen sobre la base de las evidencias científicas que alimentan los análisis de costo-efectividad, así como de las preferencias sociales y las deliberaciones éticas.

EI SPS cuenta asimismo, con un esquema legislado de financiamiento que garantiza su sustentabilidad. Este seguro se financia con contribuciones del gobierno federal, contribuciones de los gobiernos estatales y cuotas familiares, de las que están exentas las familias de menores ingresos. En 2009, el presupuesto ejercido del SPSS ascendió a 3347 millones de dólares, lo que representa un gasto anual por familia afiliada de 318 dólares ${ }^{(17)}$.

\section{EVALUACIÓN DE LA REFORMA}

El monitoreo y evaluación de los sistemas de salud es otro de los requisitos que Brachet identifica como indispensable para convertir la protección de la salud en un verdadero derecho ciudadano, ya que promueven la transparencia y la rendición de cuentas.

La reforma mexicana ha sido sujeta a evaluaciones externas muy rigurosas. La estrategia de evaluación del SPS incluyó tres componentes: i) una evaluación financiera, ii) una evaluación de procesos y iii) una evaluación de efectos ${ }^{(27)}$.

El propósito principal de la evaluación financiera es dar seguimiento a la evolución de los cinco desequilibrios financieros que dieron origen al SPSS. Los datos de dicha evaluación y de estudios posteriores indican que el gasto en salud, como porcentaje del PIB, crecerá a alrededor de $7 \%$ en 2010 como consecuencia directa de la movilización de recursos públicos adicionales vinculados con la reforma; que para ese mismo año el sistema mexicano de salud estará financiado, en su mayor parte, con fondos públicos; que se están cerrando las brechas en las asignaciones per cápita entre la población sin seguridad social (incluidos los afiliados al SPS) y la población cubierta por la seguridad social; que la desigualdad en las contribuciones estatales para financiar la salud está disminuyendo, y que la proporción de recursos públicos asignados a la inversión en infraestructura se está incrementando ${ }^{(28,29)}$.

La evaluación de procesos señala que la implantación del SPS está asociada con una mayor utilización general de servicios de salud; que los afiliados a este seguro presentan una mayor probabilidad de utilización de servicios de hospitalización por cirugías programadas, diabetes mellitus, hipertensión arterial y problemas cardiacos; que el porcentaje de surtimiento completo de recetas en unidades ambulatorias es mayor entre los afiliados al SPS que entre los no afiliados a este seguro, y que la cobertura efectiva para once intervenciones básicas se incrementó entre 2000 y $2006^{(15,30)}$.

Finalmente, el SPS se sometió a una evaluación de impacto que hizo uso de un diseño experimental. Esta evaluación, que llevaron a cabo investigadores de la Universidad Harvard y el INSP, incluyó la realización

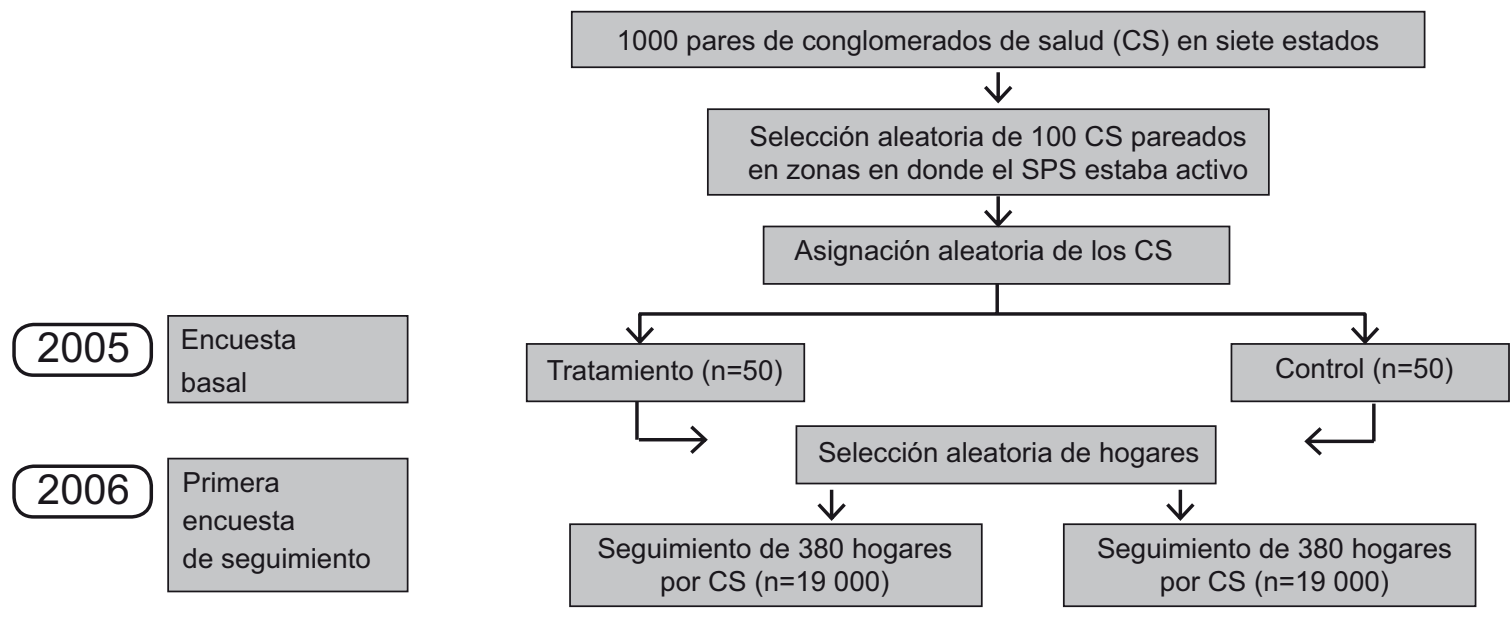

Figura 2. Diseño básico de la evaluación de impacto del Seguro Popular de Salud. 
de una encuesta en 38 mil hogares de siete entidades federativas (Figura 2). Sus resultados iniciales muestran una mejora significativa en los niveles de protección financiera en los hogares afiliados al SPS en relación con los hogares no afiliados a este seguro (31). Estas diferencias, dado el tipo de diseño utilizado, pueden atribuirse a la implantación de este instrumento de protección social en salud.

\section{CONCLUSIONES}

Son varias las lecciones que se desprenden del uso de evidencias en el diseño, implantación y evaluación en la reciente reforma al sistema mexicano de salud. La primera lección es que las evidencias incrementan considerablemente el poder de negociación de los ministros de salud, quienes de esta manera pueden convencer más fácilmente a los tomadores de decisiones, en particular a los responsables de las finanzas, de asignar más recursos a la salud.

La segunda lección de la reforma mexicana es que en el proceso de negociación e implantación de las políticas públicas son importantes tanto las evidencias técnicas, que proceden de la investigación científica, como los argumentos morales, resultado de las deliberaciones éticas.

La tercera lección es que el desarrollo de capacidades institucionales es indispensable para el éxito de las políticas públicas. En el caso mexicano, la actual reforma se benefició de 20 años de esfuerzos sostenidos para establecer y nutrir instituciones como el INSP y la FMS. Estos centros de excelencia han producido investigaciones y análisis relevantes, formado a investigadores y gerentes que han ocupado posiciones clave en el MS, llevado a cabo evaluaciones independientes y rigurosas, y mejorado la calidad de la información en salud.

La cuarta lección es que las políticas públicas pueden hacer uso de los diseños de investigación más rigurosos para ampliar el conocimiento sobre intervenciones sociales de gran envergadura. Esta reforma también ilustra la manera en que la traducción cierra el ciclo de conocimiento al generar nuevas evidencias científicas sobre lo que funciona y no funciona.

Finalmente, la reforma mexicana demuestra que el dilema entre la investigación local y global es falso. El proceso de globalización convierte el conocimiento en un bien público global que puede llevarse al centro de la agenda doméstica para enfrentar un problema local. Esta aplicación, por su parte, nutre el fondo global de experiencias, generando así un proceso de aprendizaje compartido entre países.

\section{Conflictos de Interés}

Los autores de este artículo participaron en el diseño e implantación de la reforma a la que hace referencia este trabajo.

\section{REFERENCIAS BIBLIOGRÁFICAS}

1. Ravishankar N, Gubbins P, Cooley RJ, Leach-Kemon K, Michaud CM, Jamison DT, et al. Financing of global health: tracking development assistance for health from 1990 to 2007. Lancet. 2009;373(9681):2113-24.

2. NationMaster. Health statistics: life expectancy at birth [Documento en Internet]. Woolwich: NationMaster.com. [Fecha de acceso: 25 de mayo de 2010]. Disponible en: http:// www.nationmaster.com/graph/hea_lif_exp_at_bir_tot_poplife-expectancy-birth-total-population

3. Deaton A. Health in an age of globalization Working paper 10669. Princeton, NJ: Princeton University; 2004.

4. Lozano R, Murray CJL, Frenk J, Bobadilla JL, Fernández S. El peso de la enfermedad en México: un doble reto. México, DF: Fundación Mexicana para la Salud; 1994.

5. Frenk J, Bobadilla JL, Sepúlveda J, López-Cervantes M. Health transition in middle-income countries: new challenges for health care. Health Pol Plann. 1989;4(1):29-39.

6. Cruz C, Alvarez F, Frenk J, Knaul F, Valdés C, Ramírez R. Las cuentas nacionales de salud y el financiamiento de los servicios. México, DF: Fundación Mexicana para la Salud; 1994.

7. World Health Organization (WHO). World Health Report 2000. Health systems: improving performance. Geneva: WHO; 2000.

8. Secretaría de Salud. Hogares con gastos catastróficos por motivos de salud. México, DF: Secretaría de Salud; 2001.

9. Frenk J, Knaul F, Gómez-Dantés O. Financiamiento justo y protección social universal. La reforma estructural del sistema de salud en México. México, DF: Secretaría de Salud; 2004.

10. Frenk J. Bridging the divide: global lessons from evidence-based health policy in Mexico. Lancet. 2006; 368(9539):954-61.

11. Frenk J, González-Pier E, Gómez-Dantés $O$, Lezana MA, Knaul FM. Comprehensive reform to improve health system performance in Mexico. Lancet. 2006; 368(9546):1524-34.

12. González-Pier E, Gutiérrez-Delgado C, Stevens G, Barraza-Lloréns M, Porras-Condey R, Carvalho N, et al. Priority setting for health interventions in Mexico's System for Social Protection in Health. Lancet. 2006;368(9547):1608-18.

13. Lozano R, Soliz P, Gakidou E, Abbott-Klafter J, Feehan DM, Vidal $\mathbf{C}$, et al. Benchmarking of performance of Mexican states with effective coverage. Lancet. 2006; 368(9548):1729-41.

14. Knaul FM, Arreola-Ornelas H, Méndez-Carniado $O$, Bryson-Cahn C, Barofsky J, Maguire R, et al. Evidence is good for your health system: policy reform to remedy 
catastrophic and impoverishing health spending in Mexico. Lancet. 2006; 368(9549):1828-41.

15. Gakidou E, Lozano R, González-Pier E, Abbott-Klafter J, Barofsky JT, Bryson-Cahn C, et al. Assessing the effect of the 2001-06 Mexican health reform: an interim report card. Lancet. 2006; 368(9550):1920-35.

16. Sepúlveda J, Bustreo F, Tapia R, Rivera J, Lozano R, Oláiz G, et al. Improvement of child survival in Mexico: the diagonal approach. Lancet. 2006; 368(9551):2017-27.

17. Seguro Popular de Salud. Informe de Resultados del Segundo Semestre de 2009 [Página de Internet]. México, DF: Comisión Nacional de Protección Social en Salud. [Fecha de acceso: 25 de mayo de 2010]. Disponible en: http:// www.seguro-popular.gob.mx

18. Roberts M, Hsiao W, Berman P, Reich M. Getting health reform right. A guide to improving performance and equity. New York: Oxford University Press; 2004.

19. Frenk J, Gómez-Dantés O. Ideas and ideals: ethical basis of health reform in Mexico. Lancet. 2009;373(9673):1406-8.

20. Donabedian A. Aspects of medical care administration: Specifying requirements for health care. Cambridge, Mass: Harvard University press; 1976.

21. Daniels N, Light DW, Caplan RL. Benchmarks of fairness for health care reform. New York: Oxford University Press; 2006.

22. Priester R. A values framework for health system reform. Health Affairs. 1992;11(1):84-107.

23. Frenk J, Gómez-Dantés O. La democratización de la salud. Una visión para el futuro del sistema de salud en México. Gac Med Mex. 2001;137(3):281-87.
24. O’Donnell G, Schmitter P. Transiciones desde un gobierno autoritario. Buenos Aires: Paidos; 1991.

25. Marshall TH. Class, citizenship and social development. New York: Doubleday Anchor Book; 1965.

26. Brachet-Márquez V. Ciudadanía para la salud: una propuesta. En: Uribe M, López Cervantes, editores. Reflexiones acerca de la salud en México. México DF: Médica Sur, Editorial Panamericana; 2001. p. 43-47.

27. Secretaría de Salud. Sistema de Protección Social en Salud. Estrategia de evaluación. México DF: Secretaría de Salud; 2006.

28. Secretaría de Salud. Sistema de Protección Social en Salud. Evaluación financiera. México DF: Secretaría de Salud; 2006.

29. Frenk J, Gómez-Dantés O, Knaul FM. The democratization of health in Mexico: financial innovations for universal coverage. Bull WHO. 2009;87(7):542-8.

30. Secretaría de Salud. Sistema de Protección Social en Salud. Evaluación de procesos. México DF: Secretaría de Salud; 2006.

31. King G, Gakidou E, Imai K, Lakin J, Moore RT, Nall C, et al. Public policy for the poor? A randomized assessment of the Mexican universal health insurance programme. Lancet. 2009;373(9673):1447-54.

Correspondencia: Dr. Octavio Gómez-Dantés

Dirección: 2. ${ }^{a}$ Privada Primavera N. ${ }^{\circ} 13$, Colonia Chamilpa

62219, Cuernavaca, Morelos, México.

Teléfono: (777)102-9203

Correo electrónico: ocogomez@yahoo.com

\section{Consulte las ediciones anteriores de la} Revista Peruana de Medicina Experimental y Salud Pública en www.scielo.org.pe

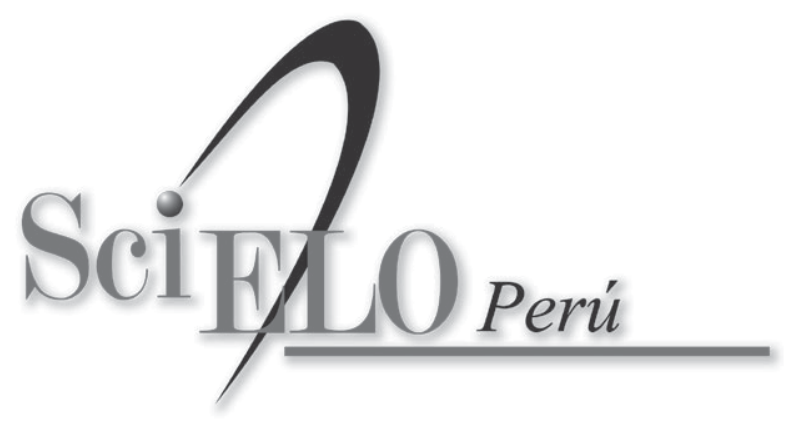

